\title{
molecules
}

ISSN 1420-3049

www.mdpi.com/journal/molecules

Review

\section{Natural Products from the Genus Tephrosia}

Yinning Chen ${ }^{1}$, Tao Yan ${ }^{2}$, Chenghai Gao ${ }^{3}$, Wenhao Cao ${ }^{2}$ and Riming Huang ${ }^{1, *}$

1 Key Laboratory of Plant Resources Conservation and Sustainable Utilization,

South China Botanical Garden, Chinese Academy of Sciences, Guangzhou 510650, China;

E-Mail: chendianyu3356@163.com

2 South China Sea Institute of Oceanology, Chinese Academy of Sciences, Guangzhou 510301, China; E-Mails: yantao@scsio.ac.cn (T.Y.); chromo@163.com (W.C.)

3 Guangxi Key Laboratory of Marine Environmental Science, Guangxi Academy of Sciences, Nanning 530007, China; E-Mail: gaochenghai@aliyun.com

* Author to whom correspondence should be addressed; E-Mail: huang_riming@hotmail.com; Tel.: +86-20-3525-2958.

Received: 10 December 2013; in revised form: 2 January 2014 / Accepted: 13 January 2014 /

Published: 27 January 2014

\begin{abstract}
The genus Tephrosia, belonging to the Leguminosae family, is a large pantropical genus of more than 350 species, many of which have important traditional uses in agriculture because they possess the bioactivity of phytoalexins. This review not only outlines the sources, chemistry and biological evaluations of natural products from the genus Tephrosia worldwide that have appeared in literature from 1910 to December 2013, but also covers work related to proposed biosynthetic pathways and synthesis of some natural products from the genus Tephrosia, with 105 citations and 168 new compounds.
\end{abstract}

Keywords: Tephrosia; chemical constituents; phytoalexins; proposed biosynthetic pathways; synthesis; biological activity

\section{Introduction}

The genus Tephrosia, belonging to the Leguminosae family, is a large pantropical genus of more than 350 species, many of which have important traditional uses [1,2]. Phytochemical investigations have revealed the presence of glucosides, rotenoids, isoflavones, chalcones, flavanones, flavanols, and prenylated flavonoids [1-9] of chemotaxonomic importance in the genus [10]. Moreover, bioactivity 
has been studied extensively, indicating that chemical constituents and extracts of the genus Tephrosia exhibited diverse bioactivities, such as insecticidal [11], antiviral [12], antiprotozoal [13], antiplasmodial [14] and cytotoxic [15] activities.

So far, the reviews on natural products isolated from the genus Tephrosia are limited [16]. To gain a comprehensive and systematic understanding of this genus, this review outlines the chemistry, proposed biosynthetic pathways, synthesis, and biological evaluations of natural products from the genus Tephrosia worldwide that have appeared in literature from 1971 to December 2013, with 105 citations and 168 new compounds from them.

\section{Chemical Constituents}

The chemical constituents of the genus Tephrosia reported since 1910 (compounds 1-168) are shown in Table 1 and Figures 1-10 below with their names, and their biological sources. As listed in the table and Figures 1-7, flavonoids are the predominant constituents of this genus.

Figure 1. Flavones from genus Tephrosia.
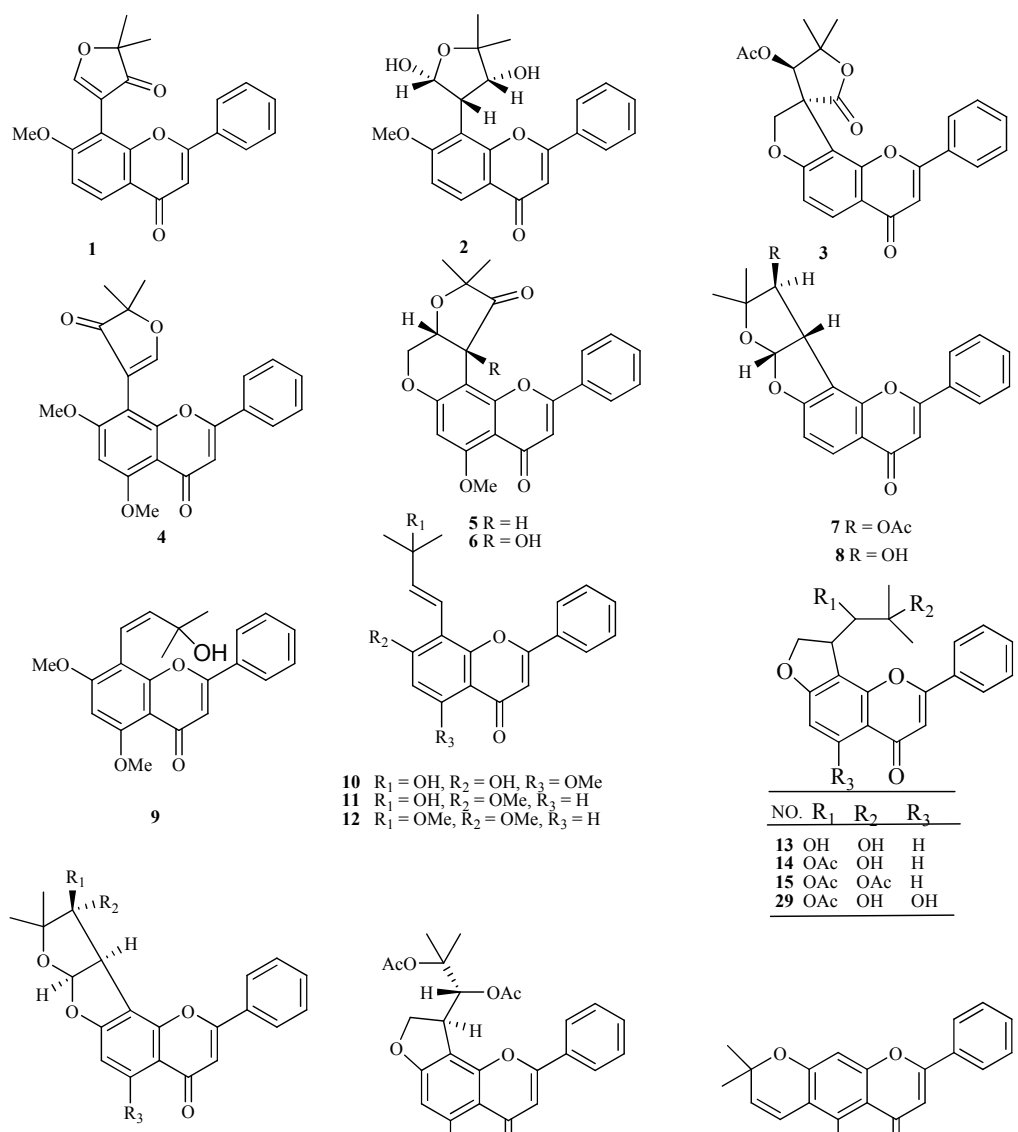

$16 \mathrm{R}_{1}=\mathrm{H}, \mathrm{R}_{2}=\mathrm{OAc}, \mathrm{R}_{3}=\mathrm{OMe}$

$17 \mathrm{R}_{1}=\mathrm{H}, \mathrm{R}_{2}=\mathrm{H}, \mathrm{R}_{3}=\mathrm{OMe}$
$18 \mathrm{R}_{1}=\mathrm{OH}, \mathrm{R}_{2}=\mathrm{H}, \mathrm{R}_{3}=\mathrm{H}$

$19 \mathrm{R}_{1}=\mathrm{OAc}, \mathrm{R}_{2}=\mathrm{H}, \mathrm{R}_{3}=\mathrm{H}$

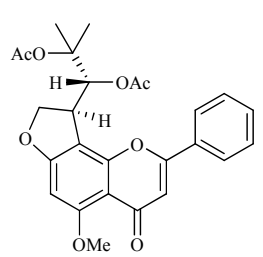

20
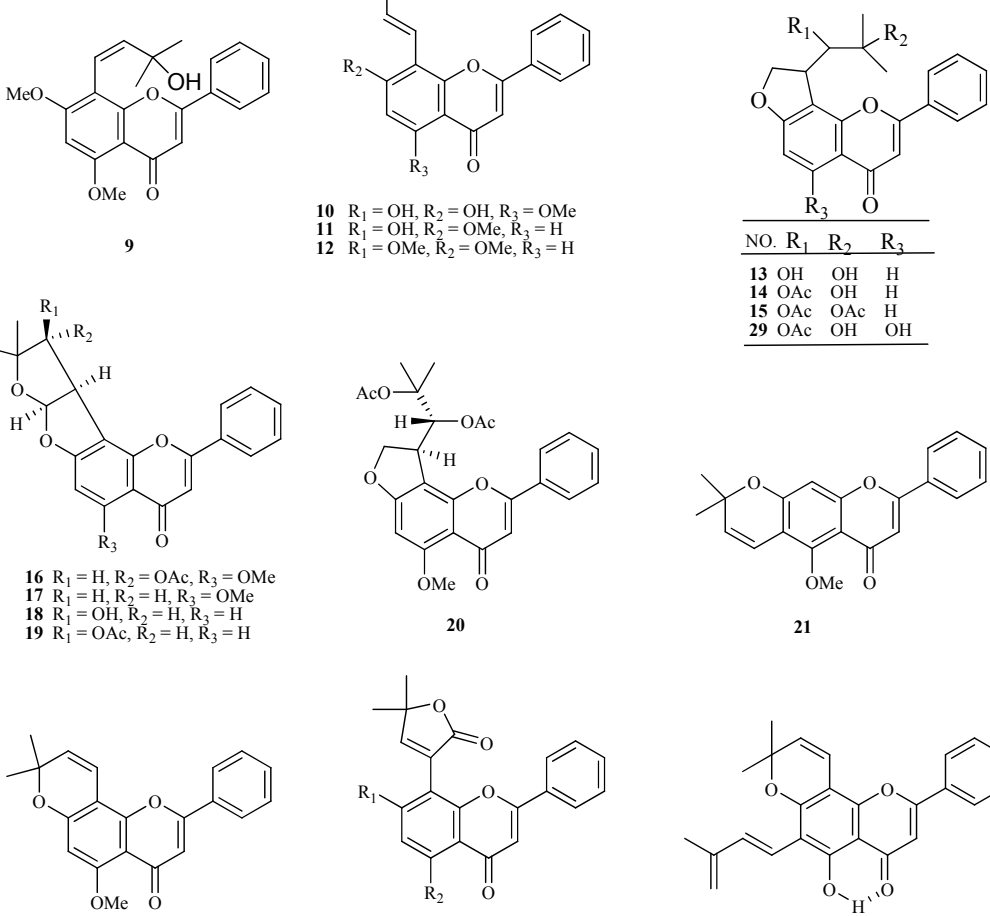

$23 \mathrm{R}_{1}=\mathrm{OMe}, \mathrm{R}_{2}=\mathrm{OMe}$

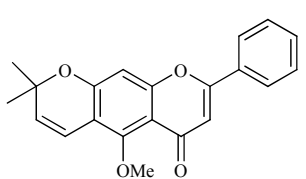

21

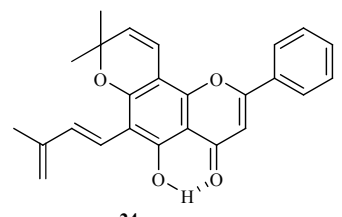


Figure 1. Cont.<smiles></smiles>

25

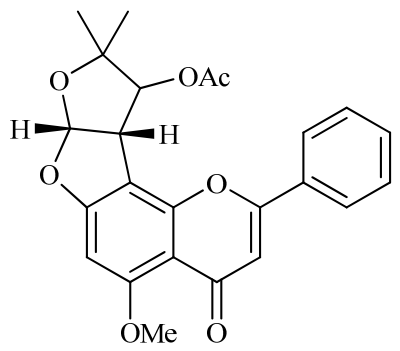

26<smiles>CC(=O)OC1C(C)(C)OC(=O)C12COc1ccc3c(=O)cc(-c4ccccc4)oc3c12</smiles>

30<smiles>CC(=O)Oc1ccc2c(=O)cc(-c3ccccc3)oc2c1[C@H]1COC(C)(C)[C@H]1OC(C)=O</smiles>

Figure 2. Flavonols from genus Tephrosia.<smiles>[R]c1cc2oc(-c3ccc(O)cc3)c([R2])c(=O)c2c(O)c1OC</smiles>

$32 \mathrm{R}_{1}=\mathrm{O}-(\alpha-\mathrm{Rha}), \mathrm{R}_{2}=\mathrm{O}-(\alpha-\mathrm{Rha}-(1 \rightarrow 2)-[\alpha-\mathrm{Rha}-(1 \rightarrow 6)]-\beta$-Gal-

$33 \mathrm{R}_{1}=\mathrm{O}-(\alpha-\mathrm{Rha}), \mathrm{R}_{2}=\mathrm{O}-(\alpha-\mathrm{Rha}-(1 \rightarrow 6)-\beta$-Gal-

$34 \mathrm{R}_{1}=\mathrm{OH}, \mathrm{R}_{2}=\mathrm{O}-(\alpha$-Rha- $(1 \rightarrow 2)-[\alpha-\mathrm{Rha}-(1 \rightarrow 6)]-\beta-$ Gal-

$35 \mathrm{R}_{1}=\mathrm{OH}, \mathrm{R}_{2}=\mathrm{O}-(\alpha$-Rha- $(1 \rightarrow 2)-[(3-O-E$-feruloyl $)-\alpha-\mathrm{Rha}-(1 \rightarrow 6)]-\beta-$ Gal-<smiles>[R]c1ccc(-c2oc3c([R])c([R])c([R])c([Y])c3c(=O)c2[R])cc1[R]</smiles>

\begin{tabular}{lllllll}
\hline NO. $\mathrm{R}_{1}$ & $\mathrm{R}_{2}$ & $\mathrm{R}_{3}$ & $\mathrm{R}_{4}$ & $\mathrm{R}_{5}$ & $\mathrm{R}_{6}$ & $\mathrm{R}_{7}$ \\
\hline 36 H & ORha & $\mathrm{OH}$ & $\mathrm{OH}$ & $\mathrm{ORha}$ & $\mathrm{OMe}$ & $\mathrm{H}$ \\
37 H & OMe & OMe & OMe & OH & OH & $\mathrm{H}$ \\
38 OMe & $\mathrm{H}$ & OMe & OH & OMe & OH & $\mathrm{H}$ \\
39 H & OEt & H & H & OH & OH & OH
\end{tabular}

Figure 3. Flavanonols from genus Tephrosia.<smiles>COc1cc2c(c3c1C(=O)[C@H](O)[C@@H](c1ccccc1)O3)C=CC(C)(C)O2</smiles>

40<smiles>[R2]c1ccc([C@H]2Oc3c(CC=C(C)C)c4c(c([R])c3C(=O)[C@@H]2[R3])C=CC(C)(C)O4)cc1</smiles>

$41 \mathrm{R}_{1}=\mathrm{OH}, \mathrm{R}_{2}=\mathrm{OH}, \mathrm{R}_{3}=\mathrm{OH}$

$42 \mathrm{R}_{1}=\mathrm{OAc}, \mathrm{R}_{2}=\mathrm{OAc}, \mathrm{R}_{3}=\mathrm{OAc}$ 
Figure 4. Flavans from genus Tephrosia.<smiles>[R3]C1C[C@@H](c2ccc(Br)cc2)Oc2c3c(cc(Br)c21)OC(C)(C)C=C3</smiles>

$43 \mathrm{R}_{1}=\mathrm{OMe}, \mathrm{R}_{2}=\mathrm{O}, \mathrm{R}_{3}=\mathrm{OH}$

$61 \mathrm{R}_{1}=\mathrm{OH}, \mathrm{R}_{2}=\mathrm{O}, \mathrm{R}_{3}=\mathrm{H}$

$62 \mathrm{R}_{1}=\mathrm{OMe}, \mathrm{R}_{2}=\mathrm{O}, \mathrm{R}_{3}=\mathrm{H}$

$63 \mathrm{R}_{1}=\mathrm{OMe}, \mathrm{R}_{2}=\mathrm{OMe}, \mathrm{R}_{3}=\mathrm{H}$<smiles>[R]c1cc(OC)c(/C=C/C(=C)C)c2c1C(=O)C[C@@H](c1ccccc1)O2</smiles><smiles>C[C@H](O)[C@H]1COC(C)(C)[C@H]1OC(=O)/C=C/c1ccccc1</smiles><smiles>[R3]c1cc(Br)c(CC=C(C)C)c2c1[C@@H]([R3])C[C@@H](c1ccccc1)O2</smiles>

$44 \mathrm{R}_{1}=\mathrm{OH}, \mathrm{R}_{2}=\mathrm{OMe}, \mathrm{R}_{3}=\mathrm{O}$ $69 \mathrm{R}_{1}=\mathrm{OMe}, \mathrm{R}_{2}=\mathrm{OH}, \mathrm{R}_{3}=\mathrm{O}$ $70 \mathrm{R}_{1}=\mathrm{OMe}, \mathrm{R}_{2}=\mathrm{OMe}, \mathrm{R}_{3}=\mathrm{H}$ $71 \mathrm{R}_{1}=\mathrm{OMe}, \mathrm{R}_{2}=\mathrm{OMe}, \mathrm{R}_{3}=\mathrm{OH}$<smiles>[R]c1ccc(C2CC(=O)c3c([R])cc(OC)c(/C=C/C(C)(C)O)c3O2)cc1</smiles>

$47 \mathrm{R}_{1}=\mathrm{OMe}, \mathrm{R}_{2}=\mathrm{H}$

$48 \mathrm{R}_{1}=\mathrm{OMe}, \mathrm{R}_{2}=\mathrm{OH}$

$77 \mathrm{R}_{1}=\mathrm{H}, \mathrm{R}_{2}=\mathrm{H}$<smiles>COc1cc2c(c3c1C(=O)C[C@H](c1ccccc1)O3)C1C(O2)C1(C)C</smiles>

45

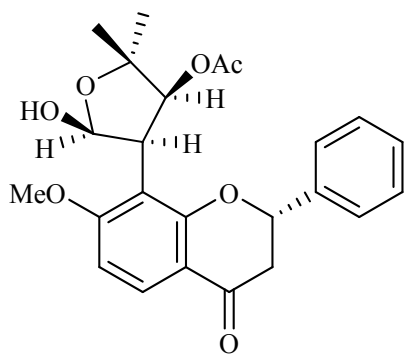

49<smiles>CC(C)=CCOc1ccc([C@H]2CC(=O)c3c(O)cc(OCC=C(C)C)cc3O2)cc1</smiles><smiles>COc1cc2c(cc1O)OC[C@H](c1cc3c(cc1O)OCO3)[C@H]2O</smiles><smiles>C=C(C)C1Cc2c(cc(OC)c3c2OC(c2ccccc2)CC3=O)O1</smiles><smiles></smiles>

54

57<smiles>[R4]c1ccc([C@H]2CC(=O)c3c(Br)c4c(c(CC=C)c3O2)OC(C)(C)C=C4)cc1</smiles>

$58 \mathrm{R}_{1}=\mathrm{R}_{2}=\mathrm{OH}$

$59 \mathrm{R}_{1}=\mathrm{R}_{2}=\mathrm{OMe}$

$60 \mathrm{R}_{1}=\mathrm{R}_{2}=\mathrm{OAc}$

$56 \mathrm{R}_{1}=\mathrm{CH}_{2} \mathrm{OAc}, \mathrm{R}_{2}=\mathrm{OH}, \mathrm{R}_{3}=\mathrm{H}$

$81 \mathrm{R}_{1}=\mathrm{OH}, \mathrm{R}_{2}=\mathrm{OMe}, \mathrm{R}_{3}=\mathrm{OMe}$

$83 \mathrm{R}_{1}=\mathrm{Me}, \mathrm{R}_{2}=\mathrm{OMe}, \mathrm{R}_{3}=\mathrm{OH}$ 
Figure 4. Cont.<smiles>C=C(C)C1Cc2c(cc(OC)c3c2O[C@@H](c2ccccc2)C=C3)O1</smiles>

65<smiles>[R]c1cc(OC)c(CCC(C)(C)O)c2c1C(=O)C[C@@H](c1ccccc1)O2</smiles>

$72 \mathrm{R}=\mathrm{OH}$

$76 \mathrm{R}=\mathrm{OMe}$<smiles>COc1cc(OC)c2c(c1)O[C@H](c1ccccc1)CC2</smiles><smiles>COc1cc(OC)c2c(c1CC1OC1(C)C)OC(c1ccccc1)CC2=O</smiles>

82<smiles>CC(C)=CCc1cc2c(c3c1OC(C)(C)C=C3)O[C@H](c1ccccc1)CC2=O</smiles><smiles>COc1cc2c(cc1C1COc3cc(O)ccc3C1)OCO2</smiles>

90<smiles>[Z]C1C[C@@H](c2ccccc2)Oc2c3c(cc(OC)c21)OC(C(=C)C)C3</smiles>

$66 \mathrm{R}=\mathrm{OMe}$

$67 \mathrm{R}=\mathrm{OH}$<smiles>COc1cc(OC)c(CC=C(C)C)c2c1C=C[C@@H](c1ccccc1)O2</smiles><smiles>COc1cc(OC)c2c(c1CC=C(C)C)OC(c1ccccc1)CC2PNC(=O)NC(C)=O</smiles><smiles>COc1cc(OC)c2c(c1CC=C(C)C)OC(C)(c1ccccc1)CC2</smiles>

74<smiles>COc1cc2c(c3c1C(O)C(O)[C@H](c1ccccc1)O3)C=CC(C)(C)O2</smiles><smiles>CC(=O)O[C@H]1[C@H]2c3c(ccc4c3O[C@H](c3ccccc3)CC4=O)O[C@H]2OC(C)(C)[C@@H]1C</smiles>

68<smiles>C=C(C)C=Cc1c(O)c2c(c3c1OC(C)(C)C=C3)O[C@@H](c1ccccc1)CC2=O</smiles>

85<smiles>C=C(C)[C@H]1Cc2c(cc(OC)c3c2O[C@@H](c2ccccc2)C[C@@H]3OC)O1</smiles><smiles>C=C(C)[C@@H]1Cc2c(cc(OC)c3c2O[C@@H](c2ccccc2)C[C@@H]3OC)O1</smiles>

89 
Figure 4. Cont.<smiles></smiles>

Figure 5. Isoflavones from genus Tephrosia.<smiles>COc1cc(-c2coc3cc(O)c(CC=C(C)C)c(O)c3c2=O)c(CC=C(C)C)c(OC)c1O</smiles><smiles>C=C(C)C1Cc2cc3c(cc2O1)OCC1c2cc4c(cc2OC31)OCO4</smiles>

94

95<smiles>[R]c1c(-c2coc3cc4c(c(O)c3c2=O)C=CC(C)(C)O4)cc(OC)c(O)c1[R]</smiles>

$96 \mathrm{R}_{1}=\mathrm{OMe}, \mathrm{R}_{2}=\mathrm{H}$ $97 \mathrm{R}_{1}=\mathrm{H}, \mathrm{R}_{2}=\mathrm{OMe}$<smiles>COCCOCCOC</smiles>
$100 \mathrm{R}=\mathrm{OH}$<smiles>[R]c1cc(-c2coc3c4c(ccc3c2=O)OCO4)ccc1OC</smiles>

98<smiles>[R]c1cc2c(cc1-c1coc3c([R])c(O)ccc3c1=O)OCO2</smiles>

$103 \mathrm{R}_{1}=\mathrm{OMe}, \mathrm{R}_{2}=\mathrm{H}$ $104 \mathrm{R}_{1}=\mathrm{OMe}, \mathrm{R}_{2}=\mathrm{OMe}$ $105 \mathrm{R}_{1}=\mathrm{H}, \mathrm{R}_{2}=\mathrm{OMe}$ 
Figure 5. Cont.<smiles>COc1cc(-c2coc3cc(O)c(CC=C(C)C)c(O)c3c2=O)c(OC)cc1O</smiles><smiles>COc1ccc(-c2coc3cc(CC=C(C)C)ccc3c2=O)cc1</smiles><smiles>C=CC(C)(C)Oc1c(OC)cc(-c2coc3cc4c(c(O)c3c2=O)C=CC(C)(C)O4)cc1OC</smiles><smiles>COc1ccc(-c2coc3cc(OCC=C(C)C)cc(OCC=C(C)C)c3c2=O)cc1</smiles><smiles>COc1cc2c(cc1OC)[C@@H]1C(=O)c3c(O)cc4c(c3O[C@H]1CO2)C=CC(C)(C)O4</smiles>

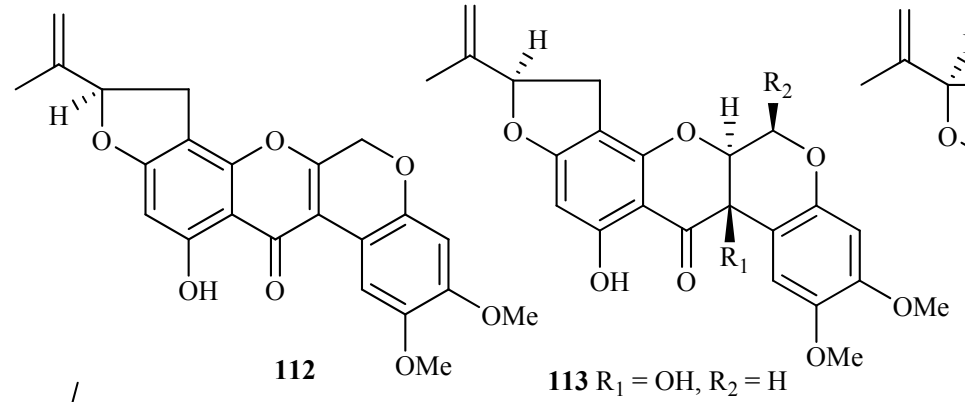<smiles>[R1]C[R]</smiles>

$\begin{array}{ll}113 \mathrm{R}_{1} & =\mathrm{OH}, \mathrm{R}_{2}=\mathrm{H} \\ 114 \mathrm{R}_{1}=\mathrm{H}, \mathrm{R}_{2}=\mathrm{OH} & \mathrm{OH}\end{array}$<smiles></smiles>

$115 \mathrm{R}_{1}=\mathrm{OH}, \mathrm{R}_{2}=\mathrm{OH}$

$117 \mathrm{R}=\mathrm{H}, \mathrm{OMe}$<smiles>CC=C(C)C</smiles><smiles>CCc1c(O)cc(O)c2c(=O)c3c(oc12)C(OC)Oc1cc(OC)c(OC)cc1-3</smiles><smiles></smiles>

$119 \mathrm{R}_{1}=\mathrm{OH}, \mathrm{R}_{2}=\mathrm{OMe}$ $120 \mathrm{R}_{1}=\mathrm{OH}, \mathrm{R}_{2}=\mathrm{OH}$

122

$121 \mathrm{R}_{1}=\mathrm{OAc}, \mathrm{R}_{2}=\mathrm{OMe}$<smiles>COc1cc2c(cc1OC(OC)OC)OCC1Oc3cc4c(c(O)c3C(=O)C21)C=CC(C)(C)O4</smiles> 
Figure5. Cont.<smiles>COc1cc2c(cc1O)OC[C@]1(O)c3cc4c(cc3O[C@@]21c1ccccc1)OCO4</smiles>

126<smiles>C=C(CO)C1Cc2cc3c(cc2O1)OCC1(O)c2cc4c(cc2OC31)OCO4</smiles><smiles></smiles>

130<smiles>C=C(C)C1Cc2cc3c(cc2O1)OC(=O)C1c2cc4c(cc2OC31)OCO4</smiles><smiles></smiles>

132<smiles>C=CC1Cc2cc3c(cc2O1)oc(=O)c1c2cc4c(cc2oc31)OCO4</smiles>

134<smiles></smiles>

Figure 6. Chalcones from genus Tephrosia.<smiles>COc1cc(O)c(/C=C/C(C)(C)O)c(O)c1C(=O)/C=C/c1ccc(O)cc1</smiles><smiles>[Z1]/C(=C\C(=O)c1ccc2occc2c1OC)c1ccc([R2])cc1</smiles>

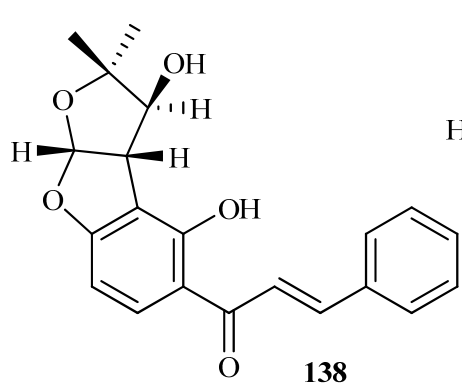

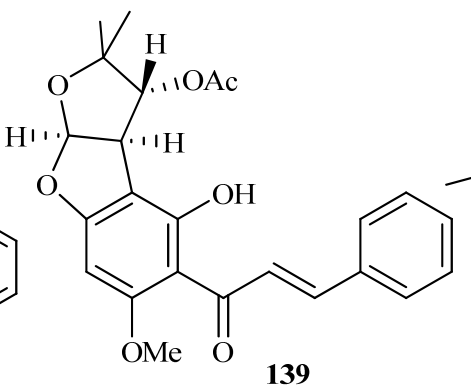
$137 \mathrm{R}_{1}=\mathrm{OMe}, \mathrm{R}_{2}=\mathrm{H}$ $142 \mathrm{R}_{1}=\mathrm{H}, \mathrm{R}_{2}=\mathrm{OMe}$

138<smiles>COc1cc2c(c(O)c1C(=O)C[C@@H](O)c1ccccc1)C=CC(C)(C)O2</smiles>

$140 \mathrm{R}_{1}=$ OMe, $\mathrm{R}_{2}=\mathrm{OMe}, \mathrm{R}_{3}=\mathrm{H}$ $143 \mathrm{R}_{1}=\mathrm{OMe}, \mathrm{R}_{2}=\mathrm{OMe}, \mathrm{R}_{3}=\mathrm{OMe}$ $144 \mathrm{R}_{1}=\mathrm{OMe}, \mathrm{R}_{2}=\mathrm{OMe}, \mathrm{R}_{3}=\mathrm{OH}$ $145 \mathrm{R}_{1}=\mathrm{OH}, \mathrm{R}_{2}=\mathrm{OMe}, \mathrm{R}_{3}=\mathrm{H}$ $148 \mathrm{R}_{1}=\mathrm{OMe}, \mathrm{R}_{2}=\mathrm{OH}, \mathrm{R}_{3}=\mathrm{H}$ $149 \mathrm{R}_{1}=\mathrm{OMe}, \mathrm{R}_{2}=\mathrm{H}, \mathrm{R}_{3}=\mathrm{OH}$ 
Figure 6. Cont.<smiles>CC(C)=CCc1c(O)c(C(=O)/C=C/c2ccccc2)cc2c1OC(C)(C)C=C2</smiles><smiles>C=C(C)C1Cc2c(cc(OC)c(C(=O)/C=C/c3ccccc3)c2O)O1</smiles><smiles>COc1cc(O)c(C(=O)/C=C/c2ccc3c(c2)OCO3)c(OC)c1</smiles><smiles>[R3]c1c(CC=C(C)C)c(O)c(CC=C(C)C)c([R3])c1C(=O)/C=C/c1ccccc1</smiles>

$151 \mathrm{R}_{1}=\mathrm{H}, \mathrm{R}_{2}=\mathrm{OH}$<smiles>COC1=CC(=O)C(CC=C(C)C)(CC=C(C)C)C(=O)C1=C(O)/C=C/c1ccccc1</smiles>

Figure 7. Other flavonoids from genus Tephrosia.<smiles>COC1=C2C=CO[C@H]2C=C/C1=C/C=C/c1ccccc1</smiles>

157<smiles>C=C(C)C1Cc2c(ccc3c2OC2(COc4cc(OC)c(OC)cc4C2=O)C3=O)OC1=O</smiles><smiles>C=C(C)C1Cc2cc(C(=O)C(=O)c3cc4c(cc3O)OCO4)c(O)cc2O1</smiles><smiles>CCOCC</smiles>

Figure 8. Triterpenoid from genus Tephrosia.<smiles>CC1(C)CCC2(C(=O)O)CCC3(C)C(=CCC4C5(C)CCC(O)C(C)(C)C5CCC43C)C2C1</smiles> 
Figure 9. Sesquiterpenes from genus Tephrosia.<smiles>C=C1CC[C@@H](O)[C@@]2(C)CC[C@H](C(C)C)[C@H](O)[C@H]12</smiles><smiles>CC(C)C1CCC2(C)C[C@H](O)[C@H](O)CC[C@]12O</smiles><smiles>C=C1CC[C@]2([13CH3])[C@@H](O)[C@H](C(C)C)[C@H](O)CC[C@]12C</smiles>

Figure 10. Other compounds from genus Tephrosia.<smiles>COc1ccc(/C=C/COC(=O)/C=C/c2ccc(OC)c(OC)c2)cc1OC</smiles><smiles>O=C(O)C[C@H](Oc1cc(O)ccc1O)c1ccccc1</smiles>

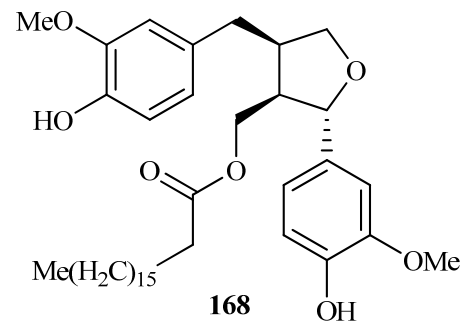

167

Table 1. Chemical constituents from the genus Tephrosia.

\begin{tabular}{|c|c|c|c|}
\hline No. & Compound class and name & Source & Ref. \\
\hline \multicolumn{4}{|c|}{ Flavones } \\
\hline 1 & tephroglabrin & T. purpurea & [3] \\
\hline 2 & tepurindiol & T. purpurea & [3] \\
\hline 3 & glabratephrin & T. apollinea & {$[10]$} \\
\hline 4 & tachrosin & Tephrosia polystachyoides & [17] \\
\hline 5 & staohyoidin & T. polystachyoides & [18] \\
\hline 6 & tephrodin & T. polystachyoides & {$[18]$} \\
\hline 7 & semiglabrin & T. semiglabra, T. apollinea & {$[19,20]$} \\
\hline 8 & semiglabrinol & T. semiglabra, T. apollinea & {$[10,19]$} \\
\hline 9 & tephrostachin & T. polystachyoides & {$[21]$} \\
\hline 10 & emoroidone & T. emoroides & {$[22]$} \\
\hline 11 & tephroapollin $\mathrm{C}$ & T. apollinea & [23] \\
\hline 12 & tephroapollin D & T. apollinea & [23] \\
\hline 13 & tephroapollin E & T. apollinea & [23] \\
\hline 14 & tephroapollin F & T. apollinea & {$[23]$} \\
\hline 15 & tephroapollin $\mathrm{G}$ & T. apollinea & [23] \\
\hline 16 & multijugin & T. multijuga & [24] \\
\hline 17 & multijuninol & T. multijuga & [24] \\
\hline 18 & pseudosemiglabrinol & T. apollinea & {$[25]$} \\
\hline 19 & $(-)$-pseudosemiglabrin & T. semiglabra & {$[26]$} \\
\hline
\end{tabular}


Table 1. Cont.

\begin{tabular}{|c|c|c|c|}
\hline No. & Compound class and name & Source & Ref. \\
\hline 20 & polystachin & T.polystachya & {$[27]$} \\
\hline 21 & 5-methoxy-6,6-dimethylpyrano[2,3:7,6]flavone & T.praecans & {$[28]$} \\
\hline 22 & candidin & T. candida & {$[29]$} \\
\hline 23 & hookerianin & T. hookeriana & {$[30]$} \\
\hline 24 & fulvinervin $\mathrm{B}$ & T. fulvinervis & {$[31]$} \\
\hline 25 & fulvinervin $\mathrm{C}$ & T. fulvinervis & {$[32]$} \\
\hline 26 & enantiomultijugin & T. viciodes & {$[33]$} \\
\hline 27 & apollinine & T. purpurea & {$[34]$} \\
\hline 28 & demethylapollinin 7-O- $\beta$-D-glucopyranoside & T. cinerea & {$[35]$} \\
\hline 29 & tephropurpulin A & T. apollinea, $T$. purpurea & {$[36,37]$} \\
\hline 30 & isoglabratephrin & T. purpurea & {$[37]$} \\
\hline 31 & terpurinflavone & T. purpurea & {$[38]$} \\
\hline \multicolumn{4}{|c|}{ Flavonols } \\
\hline 32 & 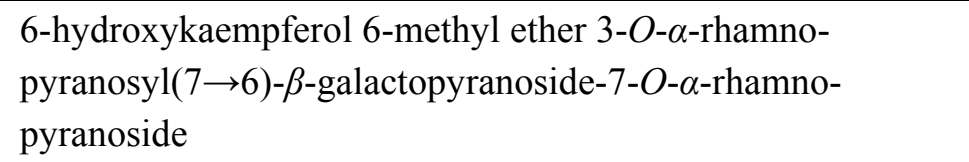 & T. vogelii & [1] \\
\hline 33 & $\begin{array}{l}\text { 6-hydroxykaempferol 6-methyl ether 3-O- } \alpha \text {-rhamno- } \\
\text { pyranosyl }(1 \rightarrow 2)[\alpha \text {-rhamnopyranosyl }(1 \rightarrow 6) \text { - } \beta \text {-galacto- } \\
\text { pyranoside }\end{array}$ & T. vogelii & {$[1]$} \\
\hline 34 & $\begin{array}{l}\text { 6-hydroxykaempferol 6-methyl ether 3-O- } \alpha \text {-rhamno- } \\
\text { pyranosyl }(1 \rightarrow 2)[\alpha \text {-rhamnopyranosyl }(1 \rightarrow 6)]-\beta \text {-galacto- } \\
\text { pyranoside-7-O- } \alpha \text {-rhamnopyranoside }\end{array}$ & T. vogelii & {$[1]$} \\
\hline 35 & $\begin{array}{l}\text { 6-hydroxykaempferol 6-methyl ether 3-O- } \alpha \text { - } \\
\text { rhamnopyranosyl }(1 \rightarrow 2)[(3-O-E \text {-feruloyl })-\alpha \text { - } \\
\text { rhamnopyranosyl }(1 \rightarrow 6)]-\beta \text {-galacto-pyranosides }\end{array}$ & T. vogelii & {$[1]$} \\
\hline 36 & 6-hydroxykaempferol 4'-methyl ether & T. candida & {$[39]$} \\
\hline 37 & candidol & & {$[40]$} \\
\hline 38 & candirone & T. candida & {$[41,42]$} \\
\hline 39 & 7-ethoxy-3,3',4'-trihydroxyflavone & T. procumbens & [43] \\
\hline \multicolumn{4}{|c|}{ Flavanonols } \\
\hline 40 & $\begin{array}{l}(2 R, 3 R)-3 \text {-hydroxy-5-methoxy-6",6"-dimethylpyrano- } \\
{[2 ", 3 ": 7,8] \text { flavanone }}\end{array}$ & T. vogelii & {$[1]$} \\
\hline 41 & lupinifolinol & T. lupinifolia & {$[44]$} \\
\hline 42 & lupinifolinol triacetate & T. lupinifolia & {$[44]$} \\
\hline \multicolumn{4}{|c|}{ Flavans } \\
\hline 43 & $\begin{array}{l}\text { (2S)-4'-hydroxy-5-methoxy-6",6"-dimethylpyrano[2",3":7,8]- } \\
\text { flavanone }\end{array}$ & T. vogelii & {$[1]$} \\
\hline 44 & (2S)-7-hydroxy-5-methoxy-8-prenylflavanone & T. vogelii & {$[1]$} \\
\hline 45 & $\begin{array}{l}(2 S)-5-\text { methoxy-6",6"-dimethy } 1-4 ", 5 \text { "-dihydrocyclopropa- } \\
{[4 ", 5 "] \text { furano[2",3":7,8]flavanone }}\end{array}$ & T. vogelii & [1] \\
\hline 46 & (2S)-5,7-dimethoxy-8-(3-methylbut-1,3-dienyl)flavanone & T. vogelii & {$[1]$} \\
\hline 47 & tephrocandidin A & T. candida & {$[2]$} \\
\hline 48 & tephrocandidin B & T. candida & {$[2]$} \\
\hline 49 & $(+)$-tephrorin A & T. purpurea & [4] \\
\hline
\end{tabular}


Table 1. Cont.

\begin{tabular}{|c|c|c|c|}
\hline No. & Compound class and name & Source & Ref. \\
\hline 50 & $(+)$-tephrorin B & T.purpurea & [4] \\
\hline 51 & (2S)-5-hydroxy-7,4'-di- $O-(\gamma, \gamma$-dimethylallyl)flavanone & T. calophylla & [6] \\
\hline 52 & 6-hydroxy-E-3-(2,5-dimethoxybenzylidine)-2',5'-dimethoxyflavanone & T. calophylla & [6] \\
\hline 53 & pumilanol & T. pumila & [13] \\
\hline 54 & emoroidenone & T. emoroides & [22] \\
\hline 55 & tephroapollin A & T. apollinea & [23] \\
\hline 56 & tephroapollin B & T. apollinea & [23] \\
\hline 57 & fulvinervin A & T. fulvinervis & [30] \\
\hline 58 & lupinifolin & T. lupinifolia & [44] \\
\hline 59 & 5,4'-O,O-dimethyl-lupinifolin & T. lupinifolia & [44] \\
\hline 60 & lupinifolin diacelate & T. lupinifolia & [44] \\
\hline 61 & obovatin & T. obovata & [45] \\
\hline 62 & obovatin methyl-ether & T. obovata & [45] \\
\hline 63 & methylhildardtol B & T. hildebrandtii & [46] \\
\hline 64 & hildgardtol B & T. hildebrandtii & [46] \\
\hline 65 & hildgardtene & T. hildebrandtii & [46] \\
\hline 66 & methylhildgardtol A & T. hildebrandtii & [46] \\
\hline 67 & hildgardtol A & T. hildebrandtii & [46] \\
\hline 68 & purpurin & T.purpurea & [47] \\
\hline 69 & tephrinone & T. villosa & [48] \\
\hline 70 & 5,7-dimethoxy-8-prenylflavan & T. madrensis & [49] \\
\hline 71 & tephrowatsin A & T. watsoniana & [50] \\
\hline 72 & tephrowatsin $\mathrm{C}$ & T. watsoniana & [50] \\
\hline 73 & tephrowatsin B & T. watsoniana & [50] \\
\hline 74 & tephrowatsin D & T. watsoniana & [50] \\
\hline 75 & tephrowatsin $\mathrm{E}$ & T. watsoniana & [50] \\
\hline 76 & nitenin & T. nitens & [51] \\
\hline 77 & falciformin & T. falciformis & [52] \\
\hline 78 & candidone & T. candida & [53] \\
\hline 79 & quercetol A & T. quercetorum & [54] \\
\hline 80 & quercetol B & T. quercetorum & [54] \\
\hline 81 & quercetol C & T. quercetorum & [54] \\
\hline 82 & 5,7-dimethoxy-8-(2,3-epoxy-3-methylbutyl)-flavanone & T. hamiltonii & [55] \\
\hline 83 & tephroleocarpin A & T. leiocarpa & [56] \\
\hline 84 & tephroleocarpin B & T. leiocarpa & [56] \\
\hline 85 & spinoflavanone A & T. spinosa & [57] \\
\hline 86 & spinoflavanone B & T. spinosa & [57] \\
\hline 87 & maxima flavanone $\mathrm{A}$ & T. maxima & [58] \\
\hline 88 & tepicanol A & T. tepicana & [59] \\
\hline 89 & crassifolin & T. crassifolia & [60] \\
\hline 90 & astraciceran & T. strigosa & [61] \\
\hline 91 & $(+)$-apollineanin & T. apollinea & [62] \\
\hline 92 & $(2 S)-5,4$ '-dihydroxy-7-O-[E-3,7-dimethyl-2,6-octadienyl]flavanone & T. villosa & [63] \\
\hline
\end{tabular}


Table 1. Cont.

\begin{tabular}{|c|c|c|c|}
\hline No. & Compound class and name & Source & Ref. \\
\hline \multicolumn{4}{|c|}{ Isoflavones } \\
\hline 93 & $\begin{array}{l}(2 S)-5,4 \text { '-dihydroxy-7- } O-[E-3,7 \text {-dimethyl-2,6-octa-dienyl }]-8-C-[E \text { - } \\
\text { 3,7-dimethyl-2,6-octadienyl]flavanone }\end{array}$ & T. villosa & {$[63]$} \\
\hline 94 & 7,4'-dihydroxy-3',5'-dimethoxyisoflavone & T. purpurea & {$[5]$} \\
\hline 95 & emoroidocarpan & T. emoroides & {$[22]$} \\
\hline 96 & elongatin & T. elongate & [64] \\
\hline 97 & pumilaisoflavone D & T. pumila & {$[65]$} \\
\hline 98 & pumilaisoflavone $\mathrm{C}$ & T.pumila & {$[65]$} \\
\hline 99 & barbigerone & T. barbigera & {$[66]$} \\
\hline 100 & 4'-demethyltoxicarol isoflavone & T. polyphylla & {$[67]$} \\
\hline 101 & maxima isoflavone $\mathrm{D}$ & T. maxima & {$[68]$} \\
\hline 102 & maxima isoflavone $\mathrm{E}$ & T. maxima & {$[68]$} \\
\hline 103 & maxima isoflavone $\mathrm{F}$ & T. maxima & {$[68]$} \\
\hline 104 & maxima isoflavone $\mathrm{G}$ & T. maxima & {$[68]$} \\
\hline 105 & viridiflorin & T. viridiflora & [69] \\
\hline 106 & maxima isoflavone $\mathrm{J}$ & T. maxima & {$[70]$} \\
\hline 107 & pumilaisoflavone A & T. pumila & [71] \\
\hline 108 & pumilaisoflavone B & T. pumila & {$[71]$} \\
\hline 109 & 7-O-geranylbiochanin A & T. tinctoria & {$[72]$} \\
\hline 110 & 5,7-di-O-prenylbiochanin A & T. tinctoria & {$[73]$} \\
\hline 111 & toxicarol & T. toxicaria & {$[74]$} \\
\hline 112 & villosinol & T. villosa & {$[75]$} \\
\hline 113 & villosol & T. villosa & {$[75]$} \\
\hline 114 & villosin & T. villoss & {$[76]$} \\
\hline 115 & villol & T. villoss & {$[76]$} \\
\hline 116 & villosone & T. villoss & {$[76]$} \\
\hline 117 & villinol & T. villoss & [76] \\
\hline 118 & dehydrodihydrorotenone & T. candida & [77] \\
\hline 119 & dihydrostemonal & T. pentaphylla & [78] \\
\hline 120 & 9-demethyldihydrostemonal & T. pentaphylla & {$[78]$} \\
\hline 121 & 6-acetoxydihydrostemonal & T. pentaphylla & [78] \\
\hline 122 & $\begin{array}{l}\text { 6a,12a-dehydro-2,3,6-trimethoxy-8-(3',3'-dimethylallyl)-9,11- } \\
\text { dihydroxyrotenone }\end{array}$ & T. villosa & [79] \\
\hline 123 & 12a-dehydro-6-hydroxysumatrol & T. villosa & {$[80]$} \\
\hline 124 & 12a-hydroxyrotenone & T. uniflora & {$[81]$} \\
\hline 125 & $12 \mathrm{a}$-hydroxy- $\beta$-toxicarol & T. candida & {$[82]$} \\
\hline 126 & tephrosol & T. villosa & {$[83]$} \\
\hline 127 & tephrocarpin & T. bidwilli & {$[84]$} \\
\hline 128 & hildecarpin & T. hildebrandtii & {$[85,86]$} \\
\hline 129 & hildecarpidin & T. hildebrandtii & {$[87]$} \\
\hline 130 & 2-methoxy-3,9-dihydroxy coumestone & T. hamiltonii & {$[88]$} \\
\hline 131 & 3,4:8,9-dimethylenedioxypterocarpan & T. aequilata & [89] \\
\hline 132 & tephcalostan & T. calophylla & {$[90]$} \\
\hline 133 & tephcalostan B & T. calophylla & [91] \\
\hline
\end{tabular}


Table 1. Cont.

\begin{tabular}{|c|c|c|c|}
\hline No. & Compound class and name & Source & Ref. \\
\hline \multicolumn{4}{|c|}{ Chalcones } \\
\hline 134 & tephcalostan C & T. calophylla & {$[91]$} \\
\hline 135 & tephcalostan D & T. calophylla & [91] \\
\hline 136 & candidachalcone & T. candida & [2] \\
\hline 137 & $O$-methylpongamol & T. purpurea & [3] \\
\hline 138 & $(+)$-tephrosone & T. purpurea & [4] \\
\hline 139 & $(+)$-tephropurpurin & T. purpurea & {$[5]$} \\
\hline 140 & 2',6'-dimethoxy-4',5'-(2"2"dimethyl)-pyranochalcone & T. pulcherrima & [7] \\
\hline 141 & $(S)$-elatadihydrochalcone & T. elata & [14] \\
\hline 142 & purpuritenin & T. purpurea & {$[15]$} \\
\hline 143 & praecansone A & T. praecans & {$[28]$} \\
\hline 144 & praecansone B & T.praecans & {$[28]$} \\
\hline 145 & obovatachalcone & T. obovata & {$[45]$} \\
\hline 146 & spinochalcone $\mathrm{C}$ & T. spinosa & [57] \\
\hline 147 & crassichalone & T. crassifolia & {$[60]$} \\
\hline 148 & oaxacacin & T. woodii & [92] \\
\hline 149 & 6'-demethoxypraecansone B & T. purpurea & [93] \\
\hline 150 & tephrone & T. candida & [94] \\
\hline 151 & spinochalcone A & T. spinosa & [95] \\
\hline 152 & spinochalcone B & T. spinosa & [95] \\
\hline 153 & 3',5'-diisopentenyl-2',4'-dihydroxychalcone & T. spinosa & {$[96]$} \\
\hline 154 & tunicatachalcone & T. tunicate & [97] \\
\hline 155 & epoxyobovatachalcone & T. carrollii & [98] \\
\hline 156 & 2',6'-dihydroxy-3'-prenyl-4'-methoxy- $\beta$-hydroxychalcone & T. major & [99] \\
\hline \multicolumn{4}{|c|}{ Other Flavonoids } \\
\hline 157 & purpureamethied & T.purpurea & {$[15]$} \\
\hline 158 & calophione A & T. calophylla & [91] \\
\hline 159 & tephrospirolactone & T. candida & [100] \\
\hline 160 & tephrospiroketone I & T. candida & [100] \\
\hline 161 & tephrospiroketone II & T. candida & [100] \\
\hline \multicolumn{4}{|c|}{ Triterpenoid } \\
\hline 162 & oleanolic acid & T. strigosa & [61] \\
\hline \multicolumn{4}{|c|}{$\begin{array}{l}\text { Sesquiterpenes } \\
\end{array}$} \\
\hline 163 & $1 \beta$-hydroxy-6,7 $\alpha$-dihydroxyeudesm-4(15)-ene & T. candida & [2] \\
\hline 164 & linkitriol & T. purpurea & [34] \\
\hline 165 & $1 \beta, 6 \alpha, 10 \alpha$-guai-4(15)-ene-6,7,10-triol & T. vogelii & [101] \\
\hline \multicolumn{4}{|c|}{ 1, } \\
\hline 166 & $\begin{array}{l}\text { 2-propenoic acid, 3-(4-(acetyloxy) -3-methoxypheny)-3(4-actyloxy)-3- } \\
\text { methoxyphenyl)-2-propenyl ester }\end{array}$ & T. purpurea & {$[34]$} \\
\hline 167 & cineroside A & T. cinerea & [35] \\
\hline 168 & $(+)$-lariciresinol-9'-stearate & T. vogelii & [101] \\
\hline
\end{tabular}

\subsection{Flavonoids}

Flavonoids were the most main constituents of the genus Tephrosia, even of the Leguminosae family. From the year of 1971, 161 flavonoids isolated from the genus Tephrosia are divided into several categories depending on their skeletons (Figures 1-7). 


\subsubsection{Flavones}

Thirty-one flavones (1-31), were isolated from T. polystachyoides, T. semiglabra, T. multijuga, T. polystachya, T. praecans, T. apollinea, T. candida, T. purpurea, T. fulvinervis, T. viciodes, T. emoroids and T. hookeriana [3,10,17-38].

\subsubsection{Flavonols}

Eight flavonols (32-39), were isolated, four, i.e., 32-34 were obtained from T. vogelii [1], one, i.e., 35-38, from T. candida [39-42] and 39 from T. procumbens [43].

\subsubsection{Flavanonols}

Only three flavanonols, 40, 41 and $\mathbf{4 2}$ were isolated from $T$. vogelii and T. lupinifolia, respectively [1,44].

\subsubsection{Flavans}

Fifty-one flavans, 43-93, were isolated from twenty-three species of the genus Tephrosia, i.e., $T$. obovata, T. villosa, T. madrensis, T. nitens, T. watsoniana, T. hildebrandtii, T. falciformis, $T$. hamiltonii, T. quercetorum, T. leiocarpa, T. spinosa, T. maxima, T. emoroides, T. tepicana, $T$. crassifolia, T. strigosa, T. pumila, T. calophylla, T. vogelii, T. apollinea, T. candida, T. purpurea and T. fulvinervis $[1,2,4,6,13,22,23,44-63]$.

\subsubsection{Isoflavones}

Forty-two isoflavones, 94-135, have been isolated and identified from this genus [5,22,64-91]. Among them, 111-125 were identified as rotenoids [74-82], 94 and 126-135 were identified as coumestan derivatives [22,83-91].

\subsubsection{Chalcones}

Twenty-one chalcones, 136-156, isolated from twelve species of genus Tephrosia, i.e., T. obovata, T. praecans, T. purpurea, T. candida, T. woodii, T. spinosa, T. crassifolia, T. tunicate, T. carrollii, T. major, T. pulcherrima and T. elata [2-5,7,14,15,28,45,57,60,92-99].

\subsubsection{Other Flavonoids}

157 was isolated from T. purpurea seeds [15]. 158 was isolated from T. calophylla [91]. 159-161 were isolated from T. candida [100].

\subsection{Triterpenoid}

Only one triterpenoid has been isolated from this genus, that is $\mathbf{1 6 2}$ from T. strigosa [61]. 


\subsection{Sesquiterpenes}

Three sesquiterpenes, 163, 164 and 165 were isolated from T. candida [2], T. purpurea [33] and T. vogelii [101], respectively.

\subsection{Others}

166-168 have been isolated from T. purpurea [34], T. cinerea [35] and T. vogelii [101], respectively.

\section{Proposed Biosynthetic Pathways and Synthesis}

8-Substituted isoflavonoids such as toxicarol isoflavone and rotenoids are well known [3]. Compounds 4-6 from $T$. polystachyoides could be explained to be evolved biogenetically from naturally occurring chrysins (A) as illustrated in the Scheme 1 [102]. It would appear that the complex substituents at C-8 arise from the ability of Tephrosia species to oxidise a 7-OMe group to a $\mathrm{O}^{+}=\mathrm{CH}_{2}$ group (Scheme 2), in the same way that closely related species of Leguminosae oxidise the 2'-OMe group of isoflavonoids to yield rotenoids [103]. A pattern that explains the various C-8 substituents in T. purpurea and T. apollinea is shown in Scheme 3. In T. polystachoides this process is taken even further and the carbon of yet another 7-OMe group is incorporated into the additional rings attached to C-7 and C-8 (Scheme 4) [3]. We could confirm the structures of compounds 7 and 8 by their conversion into semiglabrinone, isoemiglabrinone and tephroglabrin (3) as shown in Scheme 5 [3]. Purpuritenin (142) was isolated from T. purpurea has been synthetized as showed in Scheme 6 [104].

Scheme 1. Possible biogenetic pathway of compounds 4-6 of T. polystachyoides.

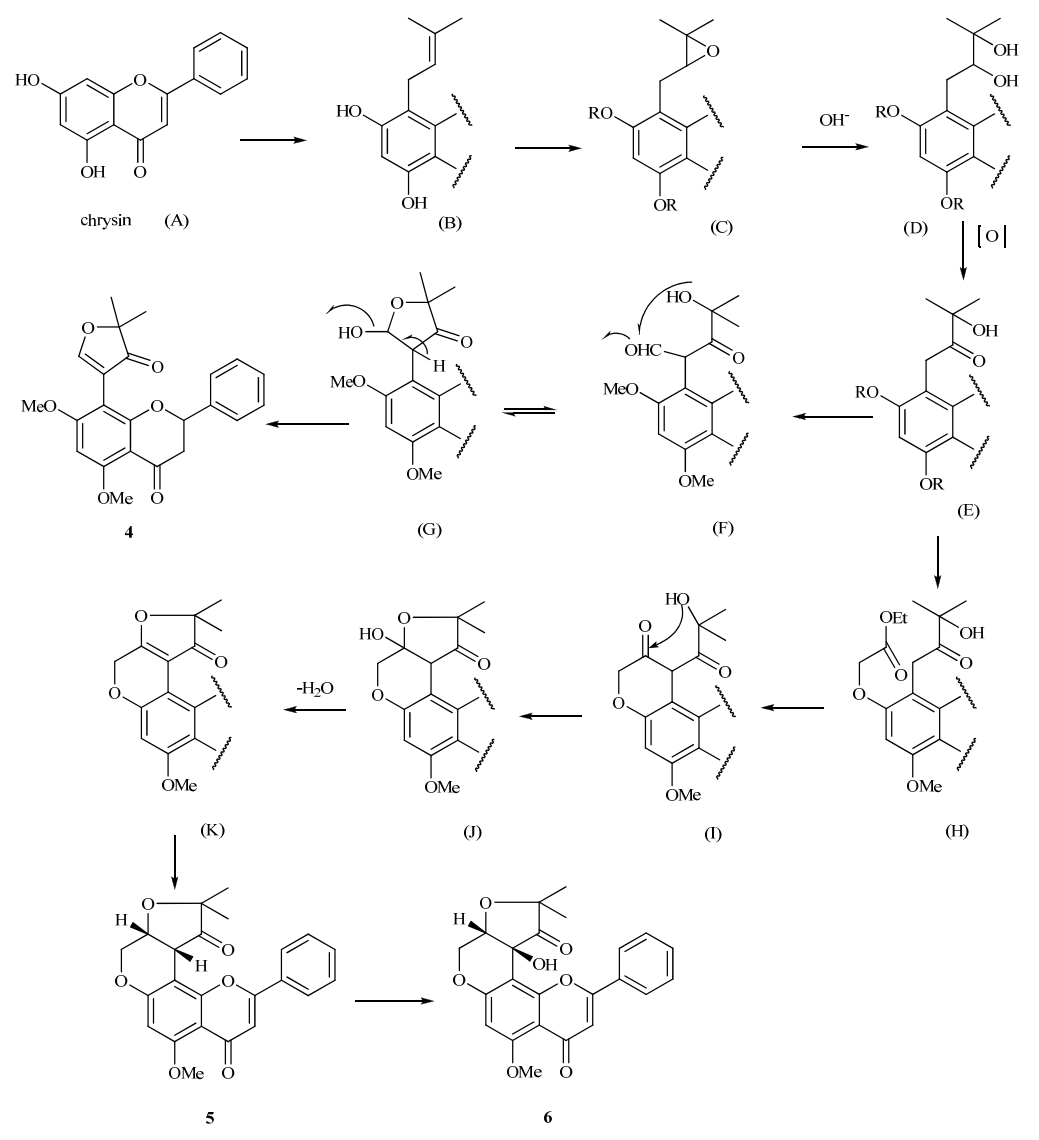


Scheme 2. Possible biogenetic pathway of compounds 8 and 11.<smiles>COc1ccc2c(=O)cc(-c3ccccc3)oc2c1/C=C/C(C)(C)O</smiles><smiles>CC1(C)O[C@H]2Oc3ccc4c(=O)cc(-c5ccccc5)oc4c3[C@H]2[C@H]1O</smiles>

8<smiles>CCc1ccc(OC)c(C)c1/C=C/C(C)(C)O</smiles><smiles>CCc1ccc2c(c1CC)C(C1OC1(C)C)CO2</smiles>

[ o ]<smiles>CCCC1c2c(ccc(CC)c2C)OC1C</smiles>

$\mathrm{H}^{+}$<smiles>O=c1cc(-c2ccccc2)oc2c1ccc1occc12</smiles>

Scheme 3. Possible biogenetic pathway of compounds 3, 8, 11, 27 and 137.

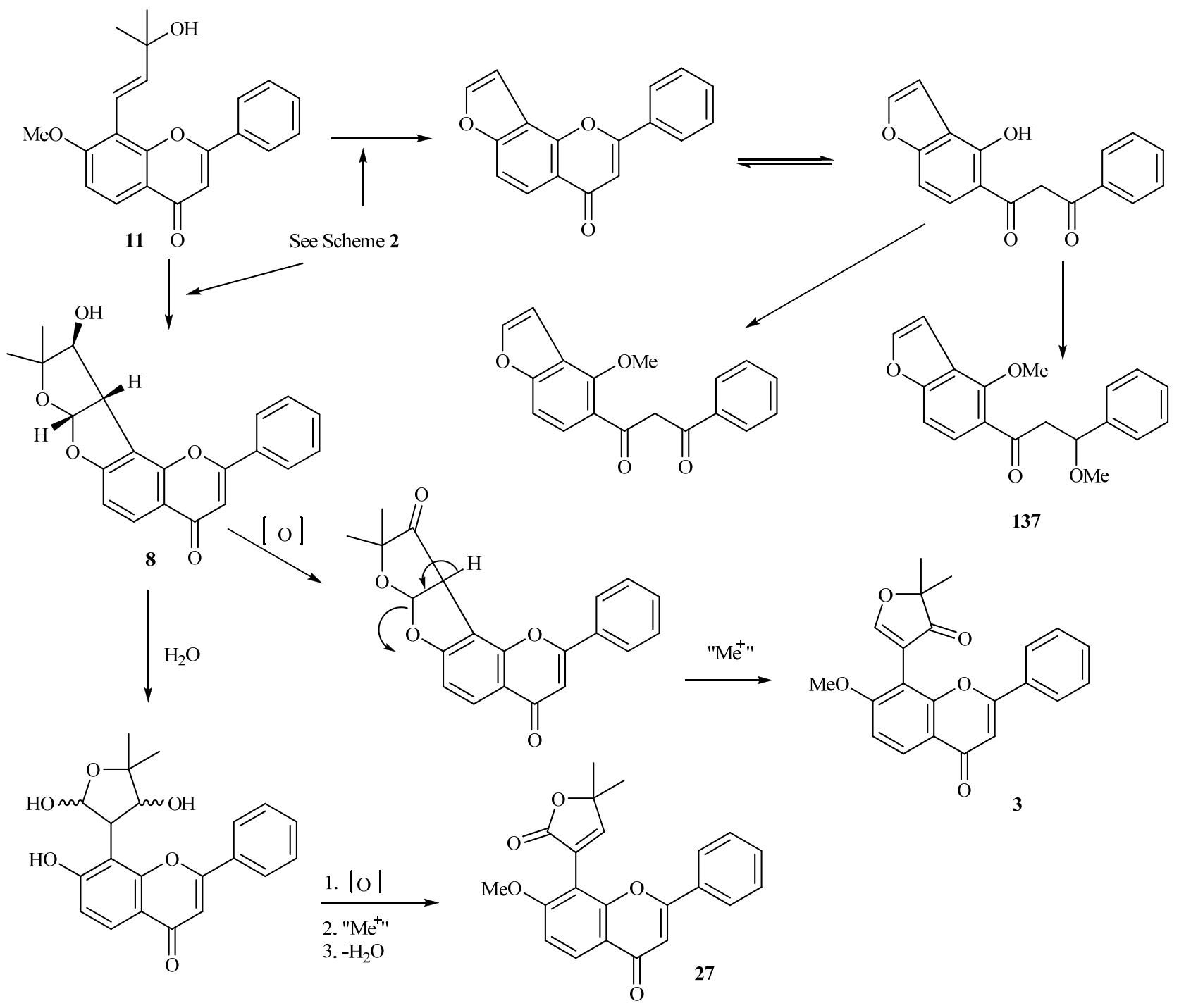


Scheme 4. Possible biogenetic pathway of compounds 4, 5 and 137.<smiles>COc1cc(OC)c2c(=O)cc(-c3ccccc3)oc2c1C1=COC(C)(C)C1=O</smiles>

Scheme 5. Transform of compounds 3 and $\mathbf{8}$.

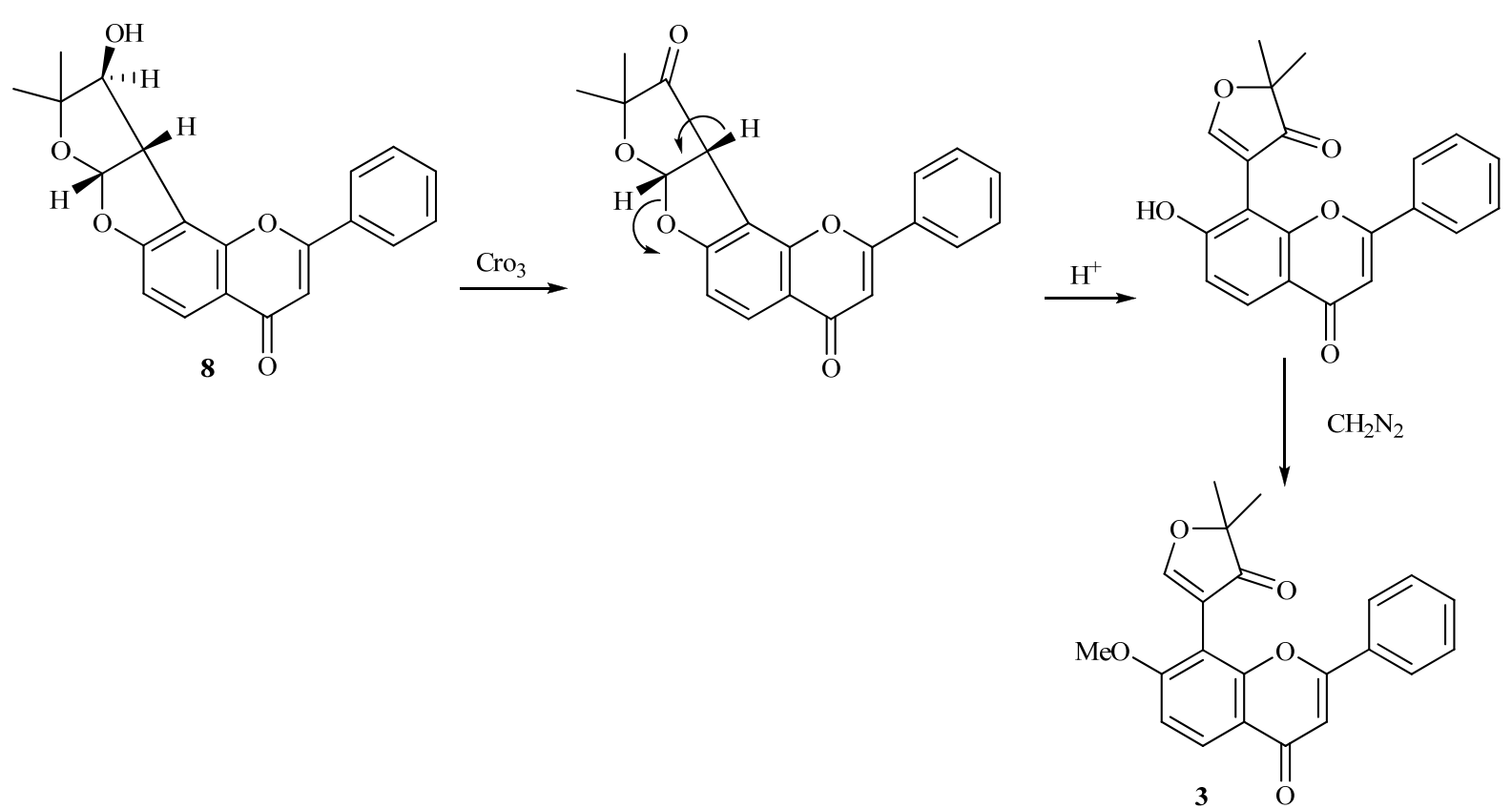


Scheme 6. The synthesis of 144.
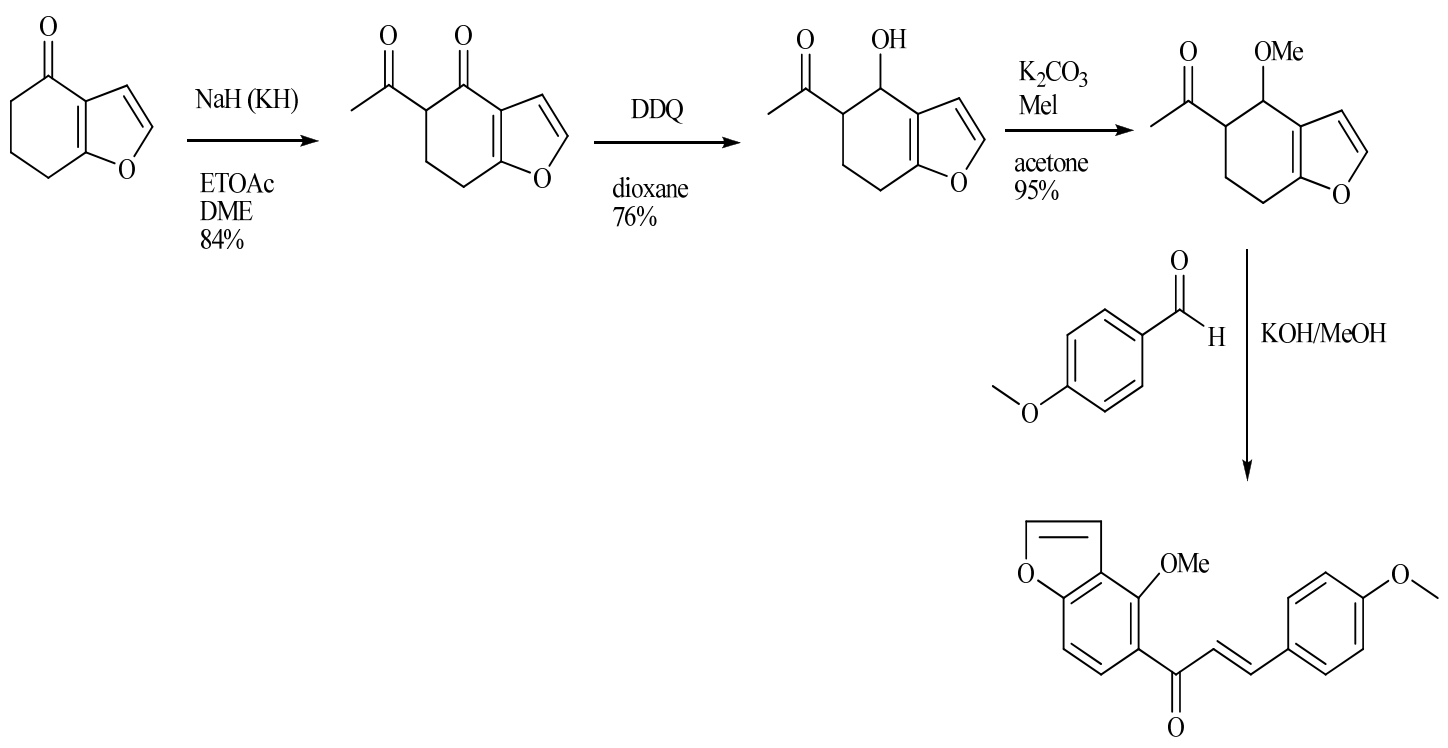

144

\section{Biological Activities}

The chemical constituents from the genus Tephrosia have been shown to exhibit various bioactivities, such as estrogenic, antitumor, antimicrobial, antiprotozoal, antifeedant activities $[2,105]$.

\subsection{Estrogenic Activity}

Candidachalcone (136) isolated from $T$. candida exhibited estrogenic activity with $\mathrm{IC}_{50}$ value of $80 \mu \mathrm{M}$, compared with $18 \mu \mathrm{M}$ for the natural steroid $17 \beta$-estradiol [2].

\subsection{Antitumor Activities}

Calophione A (158) and tephcalostans B-D (133-135) from T. calphylla were evaluated for cytotoxicity against RAW (mouse macrophage cells) and HT-29 (colon cancer cells) cancer cell lines. 158 exhibited significant cytotoxicity with $\mathrm{IC}_{50}$ of 5.00 (RAW) and $2.90 \mu \mathrm{M}$ (HT-29), respectively, while 133-135 showed moderated cytotoxicity against both RAW and HT-29 cell lines [91]. (+)-Tephrorins A (49) and B (50), and (+)-tephrosone (138) isolated from T. purpurea were evaluated for their potential cancer chemopreventive properties using a cell-based quinone reductase induction assay [4]. 7,4'-dihydroxy-3',5'-dimethoxyisoflavone (94), and (+)-tephropurpurin (139), were obtained as active compounds from $T$. purpurea, using a bioassay based on the induction of quinone reductase (QR) activity with cultured Hepa 1c1c7 mouse hepatoma cells [5].

\subsection{Antimicrobial Activities}

2',6'-Dimethoxy-4',5'-(2",2"-dimethyl)-pyranochalcone (140) from T. pulcherrima showed significant antimicrobial activity when tested against a series of micro-organisms [7]. 3,4:8,9-Dimethylenedioxypterocarpan (131) from $T$. aequilata exhibited low activity against 
gram-positive bacteria, Bacillus subtilis and Micrococcus lutea [89]. Hildecarpin (128) from T. hildebrandtii had exhibited antifungal activity against Cladosporium cucumerinum $[85,86]$.

\subsection{Antiprotozoal Activities}

Terpurinflavone (31) isolated from $T$. purpurea showed the highest antiplasmodial activity against the chloroquine-sensitive (D6) and chloroquine-resistant (W2) strains of Plasmodium falciparum with $I C_{50}$ values of $3.12 \pm 0.28 \mu \mathrm{M}$ (D6) and $6.26 \pm 2.66 \mu \mathrm{M}$ (W2) [38]. The crude extract of the seedpods of $T$. elata showed antiplasmodial activities against D6 and W2 strains of $P$. falciparum with $\mathrm{IC}_{50}$ values of $8.4 \pm 0.3$ and $8.6 \pm 1.0 \mu \mathrm{g} / \mathrm{mL}$, respectively [14]. Obovatin (61) and obovatin methyl ether (62) from $T$. obovata [45] showed antiplasmodial activities against D6 and W2 strains of $P$. falciparum with $\mathrm{IC}_{50}$ values of $4.9 \pm 1.7$ and $6.4 \pm 1.1 \mu \mathrm{g} / \mathrm{mL}$, and $3.8 \pm 0.3$ and $4.4 \pm 0.6 \mu \mathrm{g} / \mathrm{mL}$, respectively [14]. (S)-Elatadihydrochalcone (141) from $T$. elata exhibited good antiplasmodial activity against the D6 and W2 strains of $P$. falciparum with $\mathrm{IC}_{50}$ values of $2.8 \pm 0.3$ (D6) and $5.5 \pm 0.3 \mu \mathrm{g} / \mathrm{mL}$ (W2), respectively [14]. Tephcalostans C (134) and D (135) from T. calphylla were found to be weakly antiprotozoal activity in vitro [91]. Pumilanol (53) from T. pumila exhibited significant antiprotozoal activity against $T$. rhodensiense, $T$. cruzi and $L$. donovani with $\mathrm{IC}_{50}$ of $3.7,3.35$ and $17.2 \mu \mathrm{g} / \mathrm{mL}$, respectively, but displayed high toxicity towards $\mathrm{L}-6\left(\mathrm{IC}_{50}\right.$ of $\left.17.12 \mu \mathrm{g} / \mathrm{mL}\right)$ rat skeletal myoblasts [13]. Tephrinone (69) from T. villosa [48] also exhibited high degree of activity and selectivity against both T. b. rhodensiense, T. cruzi and L. donovani with $\mathrm{IC}_{50}$ of 3.3 and $16.6 \mu \mathrm{g} / \mathrm{mL}$ [13].

\subsection{Antifeedant Activities}

Emoroidenone (54) from $T$. emoroides showed strong feeding deterrent activity against Chilo partellus larvae with a mean percentage deterrence of $66.1 \%$ at a dose of $100 \mu \mathrm{g} / \mathrm{disc}$ [22]. Hildecarpin (128) from $T$. hildebrandtii had exhibited insect antifeedant activity against the legume pod-borer Maruca testulalis, and important pest of cowpea (Vigna) $[85,86]$.

\subsection{Other Activities}

(-)-Pseudosemiglabrin (19) from T. semiglabra displayed in vitro inhibitory effects on human platelet aggregation [26]. Obovatin (61), obovatin methyl-ether (62) and obovatachalcone (145) from T. obovata displayed moderate piscicidal activity against loach fish Misgurnus angullicaudatus. The TLm (median tolerance limit) values of 61, 62 and $\mathbf{1 4 5}$ were 1.25, 1.55 and 1.35 ppm, respectively [45]. Toxicarol (111) was a constituent of the South American fish poison T. toxicaria [74].

\section{Conclusions}

The genus Tephrosia, including ca. 400 species, with $c a .52$ species being investigated worldwide, was reported to possess various chemical constituents and to display diverse bioactivities, especially antiplasmodial, estrogenic, antitumor, antimicrobial, antiprotozoal, antifeedant activities. Plants of the genus Tephrosia have important traditional uses in agriculture, because they possess the bioactivity of phytoalexins. Some compounds isolated from these plants also have the bioactivity of phytoalexins according to the reported literature, which we have list in the part of the manuscript "Biological 
Activities". We think that there will be many phytoalexin-type compounds isolated from plants of the genus Tephrosia. Although the number of natural compounds was isolated from this genus, there are still many Tephrosia species that received no little attention further, phytochemical and biological studies on this genus are needed in the future. In addition, the biosynthetic pathways and synthesis of these bioactive molecules in the genus remained largely unexplored. Thus, much more chemical, biosynthetic, synthetic and biological studies should be carried out on natural compounds in Tephrosia species in order to disclose their potency, selectivity, toxicity, and availability.

\section{Acknowledgments}

We thank the authors of all the references cited herein for their valuable contributions. Financial supported for this work by grants from National Natural Science Foundation of China (No. 31100260 , 31200246), Knowledge Innovation Program of Chinese Academy of Sciences (KSCX2-EW-J-28), Program of Guangzhou City (No. 12C14061559), Foundation of Key Laboratory of Plant Resources Conservation and Sustainable Utilization, South China Botanical Garden, Chinese Academy of Sciences (No. 201210ZS).

\section{Author Contributions}

In this paper, Yinning Chen was in charge of writing the manuscript; Tao Yan was responsible for drawing the structures of the compounds; Chenghai Gao was in charge of correcting the revised manuscript; Wenhao Cao was responsible for searching for the literature; Riming Huang is the corresponding author who was responsible for arranging, checking and revising the manuscript.

\section{Conflicts of Interest}

The authors declare no conflict of interest.

\section{References}

1. Stevenson, P.C.; Kite, G.C.; Lewis, G.P.; Forest, F.; Nyirenda, S.P.; Belmain, S.R.; Sileshi, G.W.; Veitch, N.C. Distinct chemotypes of Tephrosia vogelii and implications for their use in pest control and soil enrichment. Phytochemistry 2012, 78, 135-146.

2. Hegazy, M.E.F.; Mohamed, A.E.H.; El-Halawany, A.M.; Djemgou, P.C.; Shahat, A.A.; Pare, P.W. Estrogenic activity of chemical constituents from Tephrosia candida. J. Nat. Prod. 2011, 74, 937-942.

3. Pelter, A.; Ward, R.S.; Rao, E.V.; Raju, N.R. 8-Substituted flavonoids and 3'-substituted 7-oxygenated chalcones from Tephrosia purpurea. J. Chem. Soc. Perkin Trans. 1 1981, 9, 2491-2498.

4. Chang, L.C.; Chavez, D.; Song, L.L.; Farnsworth, N.R.; Pezzuto, J.M.; Kinghorn, A.D. Absolute configuration of novel bioactive flavonoids from Tephrosia purpurea. Org. Lett. 2000, 2, 515-518.

5. Chang, L.C.; Gerhauser, C.; Song, L.; Farnsworth, N.R.; Pezzuto, J.M.; Kinghorn, A.D. Activity-guided isolation of constituents of Tephrosia purpurea with the potential to induce the phase II enzyme, quinone reductase. J. Nat. Prod. 1997, 60, 869-873. 
6. Reddy, R.V.N.; Khalivulla, S.I.; Reddy, B.A.K.; Reddy, M.V.B.; Gunasekar, D.; Deville, A.; Bodo, B. Flavonoids from Tephrosia calophylla. Nat. Prod. Commun. 2009, 4, 59-62.

7. Ganapaty, S.; Srilakshmi, G.V.K.; Pannakal, S.T.; Laatsch, H. A pyranochalcone and prenylflavanones from Tephrosia pulcherrima (Baker) drumm. Nat. Prod. Commun. 2008, 3, 49-52.

8. Kassem, M.E.S.; Sharaf, M.; Shabana, M.H.; Saleh, N.A.M. Bioactive flavonoids from Tephrosia purpurea. Nat. Prod. Commun. 2006, 1, 953-955.

9. Clarke, G.; Banerjee, S.C. A glucoside from Tephrosia purpurea. J. Chem. Soc. 1910, 97, 1833-1837.

10. Waterman, P.G.; Khalid, S.A. The major flavonoids of the seed of Tephrosia apollinea. Phytochemistry 1980, 19, 909-915.

11. Kole, R.K.; Satpathi, C.; Chowdhury, A.; Ghosh, M.R.; Adityachaudhury, N. Isolation of amorpholone, a potent rotenoid insecticide from Tephrosia candida. J. Agric. Food Chem. 1992, 40, 1208-1210.

12. Sanchez, I.; Gomez-Garibay, F.; Taboada, J.; Ruiz, B.H. Antiviral effect of flavonoids on the dengue virus. Phytother. Res. 2000, 14, 89-92.

13. Ganapaty, S.; Pannakal, S.T.; Srilakshmi, G.V.K.; Lakshmi, P.; Waterman, P.G.; Brun, R. Pumilanol, an antiprotozoal isoflavanol from Tephrosia pumila. Phytochem. Lett. 2008, 1, 175-178.

14. Muiva, L.M.; Yenesew, A.; Derese, S.; Heydenreich, M.; Peter, M.G.; Akala, H.M.; Eyase, F.; Waters, N.C.; Mutai, C.; Keriko, J.M.; et al. Antiplasmodial beta-hydroxydihydrochalcone from seedpods of Tephrosia elata. Phytochem. Lett. 2009, 2, 99-102.

15. Sinha, B.; Natu, A.A.; Nanavati, D.D. Prenylated flavonoids from Tephrosia purpurea seeds. Phytochemistry 1982, 21, 1468-1470.

16. Touqeer, S.; Saeed, M.A.; Ajaib, M. A review on the phytochemistry and pharmacology of genus Tephrosia. Phytopharmacology 2013, 4, 598-637.

17. Smalberg, T.M.; Vleggaar, R.; de Waal, H.L. Tachrosin: A new flavone from Tephrosia polystachyoides Bak F. S. Afr. J. Chem. 1971, 24, 1-8.

18. Vleggaar, R.; Smalberg, T.M.; de Waal, H.L. Two new flavones from Tephrosia polystachyoides Bakf 2. Tetrahedron Lett. 1972, 8, 703-704.

19. Smalberg, T.M.; van den Berg, A.J.; Vleggaar, R. Flavonoids from Tephrosia-VI: The structure of semiglabrin and semiglabrinol. Tetrahedron 1973, 29, 3099-3104.

20. Vleggaar, R.; Kruger, G.J.; Smalberger, T.M.; van den Berg, A.J. Flavonoids from Tephrosia. XI1. Structure of glabratephrin. Tetrahedron 1978, 34, 1405-1408.

21. Vleggaar, R.; Smalberg, T.M.; de Waal, H.L. Flavonoids from Tephrosia. V. Structure of tephrostachin. S. Afr. J. Chem. 1973, 26, 71-73.

22. Machocho, A.K.; Lwande, W.; Jondiko, J.I.; Moreka, L.V.C.; Hassanali, A. Three new flavonoids from the root of Tephrosia emoroides and their antifeedant activity against the larvae of the spotted stalk Borer Chilo-Partellus Swinhoe. Pharmaceut. Biol. 1995, 33, 222-227.

23. El-Razek, M.H.A.; Mohamed, A.E.H.H.; Ahmed, A. Prenylated flavonoids, from Tephrosia apollinea. Heterocycles 2007, 71, 2477-2490.

24. Vleggaar, R.; Smalberger, T.M.; van den Berg, A.J. Flavonoids from Tephrosia. IX. Structure of multijugin and multijuginol. Tetrahedron 1975, 31, 2571-2573. 
25. Ahmad, S. Natural occurrence of Tephrosia flavones. Phytochemistry 1986, 25, 955-958.

26. Jonathan, L.T.; Gbeassor, M.; Che, C.T.; Fong, H.H.S.; Farnsworth, N.R.; Lebreton, G.C.; Venton, D.L. Pseudosemiglabrin, a platelet-aggregation inhibitor from Tephrosia semiglabra. J. Nat. Prod. 1990, 53, 1572-1574.

27. Vleggaar, R.; Smalberger, T.M.; van Aswegen, J.L. Flavonoids from Tephrosia. X. Structure of polystachin. S. Afr. J. Chem. 1978, 31, 47-50.

28. Camele, G.; Dellemonache, F.; Dellemonache, G.; Marinibettolo, G.B. Three new flavonoids from Tephrosia praecans. Phytochemistry 1980, 19, 707-709.

29. Chibber, S.S.; Dutt, S.K. Candidin, a pyranoflavone from Tephrosia candida seeds. Phytochemistry 1981, 20, 1460-1460.

30. Prabhakar, P.; Vanangamudi, A.; Gandhidasan, R.; Raman, P.V. Hookerianin: A flavone from Tephrosia hookeriana. Phytochemistry 1996, 43, 315-316.

31. Rao, E.V.; Venkataratnam, G.; Vilain, C. Flavonoids from Tephrosia fulvinervis. Phytochemistry 1985, 24, 2427-2430.

32. Venkataratnam, G.; Rao, E.V.; Vilain, C. Fulvinervin C, a flavone from Tephrosia fulvinervis. Phytochemistry 1986, 25, 1507-1508.

33. Gomezgaribay, F.; Quijano, L.; Hernandez, C.; Rios, T. Flavonoids from Tephrosia species. IX. Enantiomultijugin, a flavone from Tephrosia viciodes. Phytochemistry 1992, 31, 2925-2926.

34. Khalafalah, A.K.; Yousef, A.H.; Esmail, A.M.; Abdelrazik, M.H.; Hegazy, M.E.; Mohamed, A.E. Chemical constituents of Tephrosia purpurea. Pharmacogn. Res. 2010, 2, 72-75.

35. Maldini, M.; Montoro, P.; Macchia, M.; Pizza, C.; Piacente, S. Profiling of phenolics from Tephrosia cinerea. Planta Med. 2011, 77, 1861-1864.

36. Khalafallah, A.K.; Suleiman, S.A.; Yousef, A.H.; El-kanzi, N.A.A.; Mohamed, A.E.H.H. Prenylated flavonoids from Tephrosia apollinea. Chin. Chem. Lett. 2009, 20, 1465-1468.

37. Hegazy, M.E.F.; Abd El-Razek, M.H.; Nagashima, F.; Asakawa, Y.; Pare, P.W. Rare prenylated flavonoids from Tephrosia purpurea. Phytochemistry 2009, 70, 1474-1477.

38. Juma, W.P.; Akala, H.M.; Eyase, F.L.; Muiva, L.M.; Heydenreich, M.; Okalebo, F.A.; Gitu, P.M.; Peter, M.G.; Walsh, D.S.; Imbuga, M.; et al. Terpurinflavone: An antiplasmodial flavone from the stem of Tephrosia purpurea. Phytochem. Lett. 2011, 4, 176-178.

39. Sarin, J.P.S.; Singh, S.; Garg, H.S.; Khanna, N.M.; Dhar, M.M. Flavonol glycoside with anticancer activity from Tephrosia candida. Phytochemistry 1976, 15, 232-234.

40. Dutt, S.K.; Chibber, S.S. Candidol, a flavonol from Tephrosia candida. Phytochemistry 1983, 22, 325-326.

41. Parmar, V.S.; Jain, R.; Simonsen, O.; Boll, P.M. Isolation of candirone-A novel pentaoxygenation pattern in a naturally-occurring 2-phenyl-4H-1-benzopyran-4-one from Tephrosia candida. Tetrahedron 1987, 43, 4241-4247.

42. Horie, T.; Kawamura, Y.; Kobayashi, T.; Yamashita, K. Revised structure of a natural flavone from Tephrosia candida. Phytochemistry 1994, 37, 1189-1191.

43. Venkataratnam, G.; Rao, E.V.; Vilain, C. Flavonoids of Tephrosia procumbens-Revised structure for praecansone A and conformation of praecansone B. J. Chem. Soc. Perkin Trans. 1 1987, 12, 2723-2727. 
44. Smalberg, T.M.; Vleggaar, R.; Weber, J.C. Flavonoids from Tephrosia. VII: Constitution and absolute-configuration of lupinifolin and lupinifolinol, two flavanones from Tephrosia lupinifolia Burch (Dc). Tetrahedron 1974, 30, 3927-3931.

45. Chen, Y.L.; Wang, Y.S.; Lin, Y.L.; Munakata, K.; Ohta, K. Obovatin, obovatin methyl-ether and obovatachalcone, new piscicidal flavonoids from Tephrosia obovata. Agric. Biol. Chem. Tokyo 1978, 42, 2431-2432.

46. Dellemonache, F.; Labbiento, L.; Marta, M.; Lwande, W. 4- $\beta$-substituted flavans from Tephrosia hildebrandtii. Phytochemistry 1986, 25, 1711-1713.

47. Gupta, R.K.; Krishnamurti, M.; Parthasarathi, J. Purpurin, a new flavanone from Tephrosia purpurea seeds. Phytochemistry 1980, 19, 1264-1264.

48. Rao, P.P.; Srimannarayana, G. Tephrinone, a new flavanone from Tephrosia villosa. Curr. Sci. India 1981, 50, 319-320.

49. Gomez, F.; Quijano, L.; Garcia, G.; Calderon, J.S.; Rios, T. A prenylated flavan from Tephrosia madrensis. Phytochemistry 1983, 22, 1305-1306.

50. Gomez, F.; Quijano, L.; Calderon, J.S.; Rodriquez, C.; Rios, T. Prenylflavans from Tephrosia watsoniana. Phytochemistry 1985, 24, 1057-1059.

51. Gomez, F.; Calderon, J.; Quijano, L.; Cruz, O.; Rios, T. Nitenin-A new flavan from Tephrosia nitens Beth. Chem. Ind. 1984, 17, 632-632.

52. Khan, H.A.; Chandrasekharan, I.; Ghanim, A. Falciformin, a flavanone from pods of Tephrosia falciformis. Phytochemistry 1986, 25, 767-768.

53. Ganguly, A.; Bhattacharyya, P.; Bhattacharyya, A.; Adityachaudhury, N. Synthesis of Candidone-A new flavanone isolated from Tephrosia candida. Indian J. Chem. B 1988, 27, 462-463.

54. Gomezgaribay, F.; Quijano, L.; Calderon, J.S.; Morales, S.; Rios, T. Flavonoids from Tephrosia species. VI. Prenylflavanols from Tephrosia quercetorum. Phytochemistry 1988, 27, 2971-2973.

55. Hussaini, F.A.; Shoeb, A. A new epoxyflavanone from Tephrosia hamiltonii. Planta Med. 1987, 2, 220-221.

56. Gomezgaribay, F.; Quijano, L.; Rios, T. Flavonoids from Tephrosia species. VII. Flavanones from Tephrosia leiocarpa. Phytochemistry 1991, 30, 3832-3834.

57. Rao, E.V.; Prasad, Y.R. Prenylated flavonoids from Tephrosia spinosa. Phytochemistry 1993, 32, $183-185$.

58. Rao, E.V.; Prasad, Y.R.; Murthy, M.S.R. A prenylated flavanone from Tephrosia maxima. Phytochemistry 1994, 37, 111-112.

59. Gomez-Garibay, F.; Calderon, J.S.; Quijano, L.; Tellez, O.; Olivares, M.D.; Rios, T. Flavonoids from Tephrosia species part 8-An unusual prenyl biflavanol from Tephrosia tepicana. Phytochemistry 1997, 46, 1285-1287.

60. Gomez-Garibay, F.; Calderon, J.S.; Arciniega, M.D.; Cespedes, C.L.; Tellez-Valdes, O.; Taboada, J. Flavonoids from Tephrosia species part 9-An unusual isopropenyldihydrofuran biflavanol from Tephrosia crassifolia. Phytochemistry 1999, 52, 1159-1163.

61. Rao, E.V.; Sridhar, P. Chemical examination of Tephrosia strigosa. Indian J. Chem. B 1999, 38, 872-873. 
62. Hisham, A.; John, S.; Al-Shuaily, W.; Asai, T.; Fujimoto, Y. (+)-Apollineanin: A new flavanone from Tephrosia apollinea. Nat. Prod. Res. 2006, 20, 1046-1052.

63. Madhusudhana, J.; Reddy, R.V.N.; Reddy, B.A.K.; Reddy, M.V.B.; Gunasekar, D.; Deville, A.; Bodo, B. Two new geranyl flavanones from Tephrosia villosa. Nat. Prod. Res. 2010, 24, 743-749.

64. Smalberger, T.M.; Vleggaar, R.; Weber, J.C. Flavonoids from Tephrosia. VIII: Structure of elongatin, an isoflavone from Tephrosia elongata E Mey. Tetrahedron 1975, 31, 2297-2301.

65. Yenesew, A.; Dagne, E.; Waterman, P.G. Flavonoids from the seed pods of Tephrosia pumila. Phytochemistry 1989, 28, 1291-1292.

66. Vilain, C. Barbigerone, a new pyranoisoflavone from seeds of Tephrosia barbigera. Phytochemistry 1980, 19, 988-989.

67. Dagne, E.; Mammo, W.; Sterner, O. Flavonoids of Tephrosia polyphylla. Phytochemistry 1992, 31, 3662-3663.

68. Rao, E.V.; Murthy, M.S.R.; Ward, R.S. Nine isoflavones from Tephrosia maxima. Phytochemistry 1984, 23, 1493-1501.

69. Gomez, F.; Calderon, J.S.; Quijano, L.; Dominguez, M.; Rios, T. Viridiflorin, an isoflavone from Tephrosia viridiflora. Phytochemistry 1985, 24, 1126-1128.

70. Murthy, M.S.R.; Rao, E.V. Maxima isoflavone J: A new O-prenylated isoflavone from Tephrosia maxima. J. Nat. Prod. 1985, 48, 967-968.

71. Dagne, E.; Dinku, B.; Gray, A.I.; Waterman, P.G. Pumilaisoflavone A and Pumilaisoflavone B from the seed pods of Tephrosia pumila. Phytochemistry 1988, 27, 1503-1505.

72. Reddy, B.A.K.; Khalivulla, S.I.; Gunasekar, D. A new prenylated isoflavone from Tephrosia tinctoria. Indian J. Chem. B 2007, 46, 366-369.

73. Khalivulla, S.I.; Reddy, B.A.K.; Gunasekar, D.; Blond, A.; Bodo, B.; Murthy, M.M.; Rao, T.P. A new di-O-prenylated isoflavone from Tephrosia tinctoria. J. Asian Nat. Prod. Res. 2008, 10, 953-955.

74. Clark, E.P. Toxicarol. A constituent of the South American fish poison Cracca (Tephrosia) toxicaria. J. Am. Chem. Soc. 1930, 52, 2461-2464.

75. Sarma, P.N.; Srimannarayana, G.; Rao, N.V.S. Constitution of villosol and villosinol, two new rotenoids from Tephrosia villosa (Linn) pods. Indian J. Chem. B 1976, 14, 152-156.

76. Krupadanam, G.L.D.; Sarma, P.N.; Srimannarayana, G.; Rao, N.V.S. New C-6 oxygenated rotenoids from Tephrosia villosa-Villosin, villosone, villol and villinol. Tetrahedron Lett. 1977, 24, 2125-2128.

77. Roy, M.; Bhattacharya, P.K.; Pal, S.; Chowdhuri, A.; Adityachaudhury, N. Dehydrodihydrorotenone and flemichapparin B in Tephrosia candida. Phytochemistry 1987, 26, 2423-2424.

78. Dagne, E.; Yenesew, A.; Waterman, P.G. Flavonoids and isoflavonoids from Tephrosia fulvinervis and Tephrosia pentaphylla. Phytochemistry 1989, 28, 3207-3210.

79. Prashant, A.; Krupadanam, G.L.D. A new prenylated dehydrorotenoid from Tephrosia villosa seeds. J. Nat. Prod. 1993, 56, 765-766.

80. Prashant, A.; Krupadanam, G.L.D. Dehydro-6-hydroxyrotenoid and lupenone from Tephrosia villosa. Phytochemistry 1993, 32, 484-486.

81. Abreu, P.M.; Luis, M.H. Constituents of Tephrosia uniflora. Nat. Prod. Lett. 1996, 9, 81-86. 
82. Andrei, C.C.; Viera, P.C.; Fernandes, J.B.; daSilva, M.F.D.F.; Fo, E.R. Dimethylchromene rotenoids from Tephrosia candida. Phytochemistry 1997, 46, 1081-1085.

83. Rao, P.P.; Srimannarayana, G. Tephrosol, a new coumestone from the roots of Tephrosia villosa. Phytochemistry 1980, 19, 1272-1273.

84. Ingham, J.L.; Markham, K.R. Tephrocarpin, a pterocarpan phytoalexin from Tephrosia bidwilli and a structure proposal for acanthocarpan. Phytochemistry 1982, 21, 2969-2972.

85. Lwande, W.; Bentley, M.D.; Hassanali, A. The structure of hildecarpin, an insect antifeedant 6a-hydroxypterocarpan from the roots of Tephrosia hildebrandtii Vatke. Int. J. Trop. Insect Sci. 1986, 7, 501-503.

86. Lwande, W.; Hassanali, A.; Njoroge, P.W.; Bentley, M.D.; Delle Monache, F.; Jondiko, J.I. A new 6a-hydroxypterocarpan with insect antifeedant and antifungal properties from the roots of Tephrosia hildebrandtii Vatke. Int. J. Trop. Insect Sci. 1985, 6, 537-541.

87. Lwande, W.; Bentley, M.D.; Macfoy, C.; Lugemwa, F.N.; Hassanali, A.; Nyandat, E. A new pterocarpan from the roots of Tephrosia hildebrandtii. Phytochemistry 1987, 26, 2425-2426.

88. Rajani, P.; Sarma, P.N. A coumestone from the roots of Tephrosia hamiltonii. Phytochemistry 1988, 27, 648-649.

89. Tarus, P.K.; Machocho, A.K.; Lang'at-Thoruwa, C.C.; Chhabra, S.C. Flavonoids from Tephrosia aequilata. Phytochemistry 2002, 60, 375-379.

90. Kishore, P.H.; Reddy, M.V.B.; Gunasekar, D.; Murthy, M.M.; Caux, C.; Bodo, B. A new coumestan from Tephrosia calophylla. Chem. Pharm. Bull. (Tokyo) 2003, 51, 194-196.

91. Ganapaty, S.; Srilakshmi, G.V.K.; Pannakal, S.T.; Rahman, H.; Laatsch, H.; Brun, R. Cytotoxic benzil and coumestan derivatives from Tephrosia calophylla. Phytochemistry 2009, 70, 95-99.

92. Dominguez, X.A.; Tellez, O.; Ramirez, G. Mixtecacin, a prenylated flavanone and oaxacacin its chalcone from the roots of Tephrosia woodii. Phytochemistry 1983, 22, 2047-2049.

93. Rao, E.V.; Raju, N.R. Two flavonoids from Tephrosia purpurea. Phytochemistry 1984, 23, 2339-2342.

94. Chibber, S.S.; Dutt, S.K. Tephrone, a new chalcone from Tephrosia candida seeds. Curr. Sci. India 1982, 51, 933-934.

95. Rao, E.V.; Prasad, Y.R. Two chalcones from Tephrosia spinosa. Phytochemistry 1992, 31, 2121-2122.

96. Sharma, V.M.; Rao, P.S. A prenylated chalcone from the roots of Tephrosia spinosa. Phytochemistry 1992, 31, 2915-2916.

97. Andrei, C.C.; Ferreira, D.T.; Faccione, M.; de Moraes, L.A.B.; de Carvalho, M.G.; Braz, R. C-prenylflavonoids from roots of Tephrosia tunicata. Phytochemistry 2000, 55, 799-804.

98. Gomez-Garibay, F.; Arciniega, M.D.O.; Cespedes, C.L.; Taboada, J.; Calderon, J.S. Chromene chalcones from Tephrosia carrollii and the revised structure of oaxacacin. Z. Naturforsch. C 2001, 56, 969-972.

99. Gomez-Garibay, F.; Tellez-Valdez, O.; Moreno-Torres, G.; Calderon, J.S. Flavonoids from Tephrosia major. A new prenyl- $\beta$-hydroxychalcone. Z. Naturforsch. $C$ 2002, 57, 579-583.

100. Andrei, C.C.; Vieira, P.C.; Fernandes, J.B.; da Silva, M.F.; Rodrigues Fo, E. New spirorotenoids from Tephrosia candida. Z. Naturforsch. C 2002, 57, 418-422. 
101. Wei, H.H.; Xu, H.H.; Xie, H.H.; Xu, L.X.; Wei, X.Y. Sesquiterpenes and lignans from Tephrosia vogelii. Helv. Chim. Acta 2009, 92, 370-374.

102. Jain, A.C.; Gupta, R.C., Possible biogenesis of novel type of flavones from Tephrosia polystachyoides. Curr. Sci. India 1978, 47, 770-770.

103. Crombie, L.; Dewick, P.M.; Whiting, D.A. Biosynthesis of rotenoids - Chalcone, isoflavone, and rotenoid stages in formation of amorphigenin by Amorpha fruticosa seedlings. J. Chem. Soc. Perkin Trans. 1 1973, 12, 1285-1294.

104. Lee, Y.R.; Morehead, A.T. A new route for the synthesis of furanoflavone and furanochalcone natural products. Tetrahedron 1995, 51, 4909-4922.

105. Belmain, S.R.; Amoah, B.A.; Nyirend, S.P.; Kamanula, J.F. Stevenson, P.C. Highly variable insect control efficacy of Tephrosia vogelii Chemotypes. J. Agric. Food Chem. 2012, 60, 10055-10063.

(C) 2014 by the authors; licensee MDPI, Basel, Switzerland. This article is an open access article distributed under the terms and conditions of the Creative Commons Attribution license (http://creativecommons.org/licenses/by/3.0/). 